\title{
Aligned planar-wire zero-index metamaterial for terahertz frequency range
}

\author{
E.A. Litvinov ${ }^{1}$, P. S. Demchenko ${ }^{1}$, E.B. Sheklanova ${ }^{2}$, M.K. Khodzitsky ${ }^{1}$ \\ ${ }^{1}$ Terahertz Biomedicine Laboratory, Saint-Petersburg, Russia, thzbiomedicine@corp.ifmo.ru \\ ${ }^{2}$ International Laboratory "Nonlinear optical molecular crystals and microlasers", Saint-Petersburg, Russia
}

\section{Introduction}

Demonstrations of specific class of materials with extremely small (near zero) values of permittivity have attracted attention of scientific community ${ }^{1}$. These unique materials have the refractive index equal to zero what causes almost constant spatial distribution of the electric field of the propagating wave inside these media ${ }^{2}$.

Considering its extreme properties, ENZ-material can also be used as delay line that brings no phase delay in propagating wave ${ }^{3}$, as ideal coupler between waveguides of the same diameter and can find effective application in cloaking 4 . It should be mentioned that ENZ-, MNZ- ("mu near zero") and LH- ("left-handed") materials can be designed using transformation optics approximation ${ }^{5-7}$.

ENZ or MNZ coating may be used to radically enhance transmission through subwavelength aperture in planar ideally opaque screen which allow to overcome the diffraction limit. In general, isolated subwavelength aperture can't provide enough transfer of energy, but with ENZ coating the transfer increases by several orders of magnitude ${ }^{8}$.

This work is dedicated to zero-index (ZI) terahertz metamaterial designed with one-layer aligned planar aluminum wires on dielectric PET substrate and acting like epsilon-near-zero (ENZ) medium.

Metal stripes on dielectric substrate can be easily manufactured. The idea is that metal stripes may have permittivity described by Drude model in terahertz frequency range, what gives the opportunity to design ENZmaterial.

\section{Numerical simulation of the structure}

To prove that such structure can act like ENZ-material, number of numerical simulations was done with the periodic boundary conditions. Simulations were done also in two steps: the first is for the unit cell and the second is for the one-layered structure.

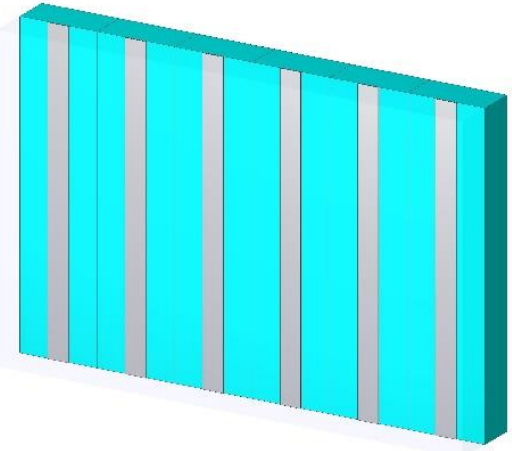

Fig. 1. The investigated structure: dielectric PET (Polyethylene terephthalate) substrate and parallel aluminum stripes.

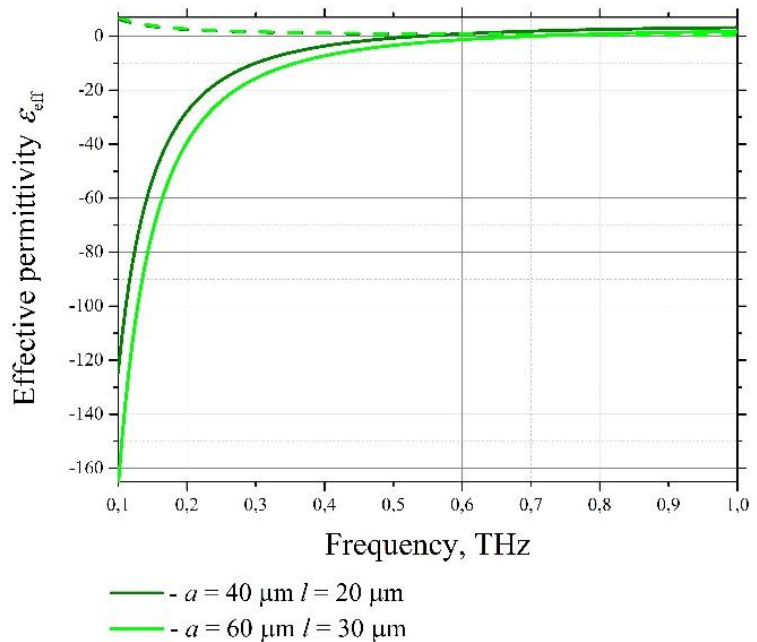

(a)

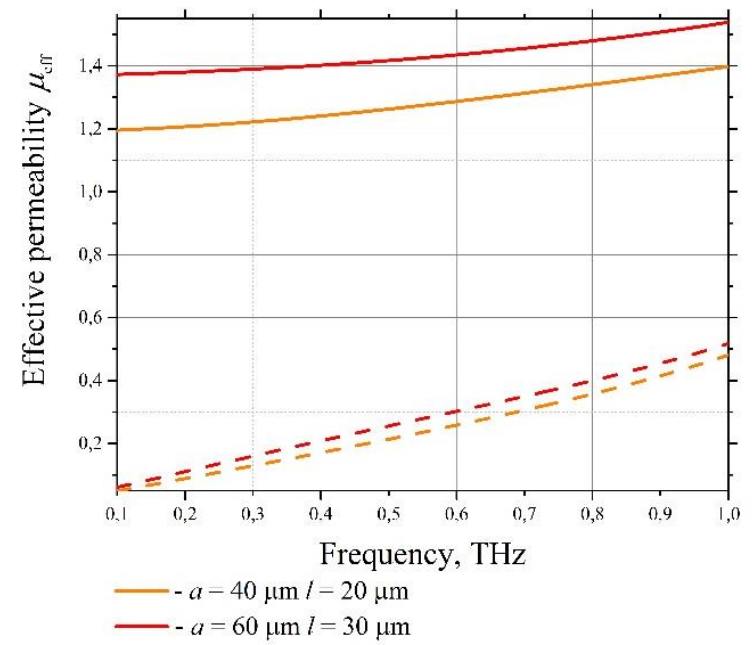

(b)

Fig. 1. Results of numerical simulations of the striped metamaterial ( $a$ is the lattice constant, $l$ is the width of the stripe). 'Electric' plasma frequency for $l=20 \mu \mathrm{m}$ is $0,641 \mathrm{THz}$ and for $l=30 \mu \mathrm{m}$ is $0,562 \mathrm{THz}$.

\section{Experimental verification}

The material was made by laser ablation method using LaserGraver LG2500 SP47. Two samples were used for the experiment: ones with $l=20 \mu \mathrm{m} \quad a=40 \mu \mathrm{m}$ and $l=30 \mu \mathrm{m}$ $a=60 \mu \mathrm{m}$. Examined samples were measured using timedomain terahertz spectroscopy in $0,1-1 \mathrm{THz}$ frequency range in transmission mode. 


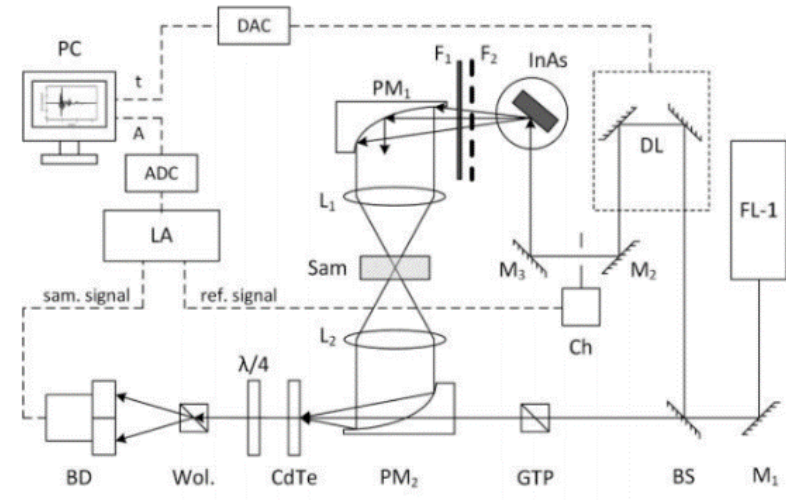

Figure. 1. Experimental $\mathrm{THz}$ TDS setup ${ }^{9}$ in transmission mode (FL-1 - femtosecond laser based on potassium-yttrium tungstate crystal activated with ytterbium (Yb: KYW), generating femtosecond pulses; F1,2 - teflon filters for IR wavelength range cutting off, BS - beamsplitter, DL - optical delay line, M1,2,3 mirrors, Sam - investigated sample, Wol. - Wollaston prism, CdTe - electro-optical cadmium-telluric crystal, BD - balanced detector, LA - lock-in amplifier, PC - personal computer, GTP Glan-Taylor prism, PM1,2 - parabolic mirrors, Ch - chopper, DAC - digital to analog converter, ADC - analog to digital converter)

The experiment showed that samples have the dispersion of refractive index according to Drude model (Figure 8). In a little area near plasma frequencies samples behave as ENZ-material. It should be mentioned that below plasma frequencies medium shows left-handed properties in materials previously designed ${ }^{10,11}$.

(a)

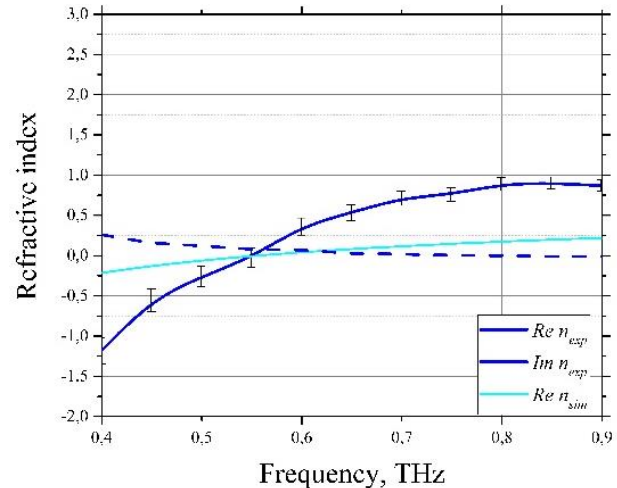

(b)

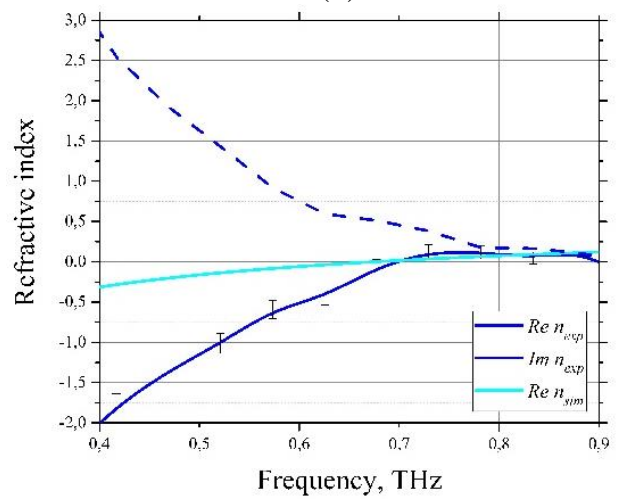

Figure 2. Experimental (blue) and calculated (cyan) effective permittivity and permeability of two striped metamaterial samples. (a) is the data on s ample with $l=20 \mu \mathrm{m} a=40 \mu \mathrm{m}$ and (b) is the data on sample with $l=30 \mu \mathrm{m} a=60 \mu \mathrm{m}$. Plasma frequencies are approximately $0,556 \mathrm{THz}$ for (a) and $0,648 \mathrm{THz}$ for (b).

\section{Conclusion}

This paper showed the possibility of designing ZImaterials for terahertz frequency range using one-layered copper wire or aluminum stripe lattices. Copper-wired medium can be manufactured using electrochemical etching or electron-beam lithography. Aluminum-striped material was made from metallized PET substrate using laser ablation. ENZ properties of metal-striped materials were showed using numerical simulation and verified experimentally. Two striped-material samples were about to have effective plasma frequency in terahertz frequency range and showed left-handed properties below the plasma frequency.

\section{References}

[1] Engheta, N. and Liberal, I., "The rise of near-zero technologies," Science 358(6370), 1540-1541 (2017).

[2] Ziolkowski, R. W., "Propagation in and scattering from a matched metamaterial having a zero index of refraction," Physical Review E 70(4) (2004).

[3] Enoch, S., Tayeb, G., Sabouroux, P., Guérin, N. and Vincent, P., "A Metamaterial for Directive Emission," Physical Review Letters 89(21) (2002).

[4] Alu, A., Engheta, N., Erentok, A. and Ziolkowski, R., "Single-Negative, Double-Negative, and Low-index Metamaterials and their Electromagnetic Applications," IEEE Antennas and Propagation Magazine 49(1), 23-36 (2007).

[5] Islam, S. S., Faruque, M. R. I. and Islam, M. T., "An Object-Independent ENZ Metamaterial-Based Wideband Electromagnetic Cloak," Scientific Reports 6(1) (2016).

[6] Javani, M. H. and Stockman, M. I., "Real and Imaginary Properties of Epsilon-Near-Zero Materials," Physical Review Letters 117(10) (2016).

[7] Kyoung, J., Park, D. J., Byun, S. J., Lee, J., Choi, S. B. and Park, S., "Epsilon-Near-Zero meta-lens for high resolution wide-field imaging," Optics Express 22(26), 31875 (2014).

[8] Shi, K. and Lu, Z., "Field-effect optical modulation based on epsilon-near-zero conductive oxide," Optics Communications 370, 22-28 (2016).

[9] Bespalov, V. G., Gorodetskiǔ, A. A., Denisyuk, I. Y., Kozlov, S. A., Krylov, V. N., Lukomskiı̌, G. V., Petrov, N. V. and Putilin, S. é., "Methods of generating superbroadband terahertz pulses with femtosecond lasers," Journal of Optical Technology 75(10), 636 (2008).

[10] Khodzitsky, M. K., Tarapov, S. I., Belozorov, D. P., Pogorily, A. M., Tovstolytkin, A. I., Belous, A. G. and Solopan, S. A., "Negative permittivity and lefthanded behavior of doped manganites in millimeter waveband," Applied Physics Letters 97(13), 131912 (2010).

[11] Khodzitsky, M. K., Kharchenko, A. A., Strashevskyi, A. V. and Tarapov, S. I., "Left-Handed Properties of Metal-Ferrite Composites Placed into Waveguide in Millimetric Wave Range," Telecommunications and Radio Engineering 68(7), 561-566 (2009). 